BRAVZULIAN JOURNAL

OF MEDICAL AND BIOLOGICAL RESH.ARCH

www.bjournal.com.br
ISSN 0100-879X

Volume 43 (5) 381-496 May 2011

BIOMEDICAL SCIENCES

AND

CLINICAL INVESTIGATION

Braz J Med Biol Res, May 2011, Volume 44(5) 489-496

doi: 10.1590/S0100-879X2011007500052

The inhibitory effect of photodynamic therapy and of an anti-VCAM-1 monoclonal antibody on the in vivo growth of C6 glioma xenografts

Qi Zhan, Wu Yue and Shaoshan $\mathrm{Hu}$

The Brazilian Journal of Medical and Biological Research is partially financed by

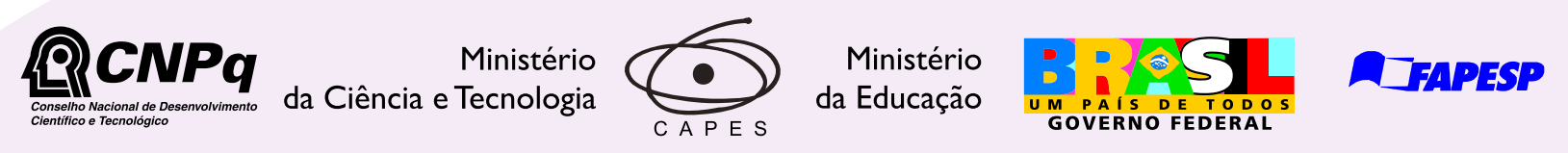

Institutional Sponsors
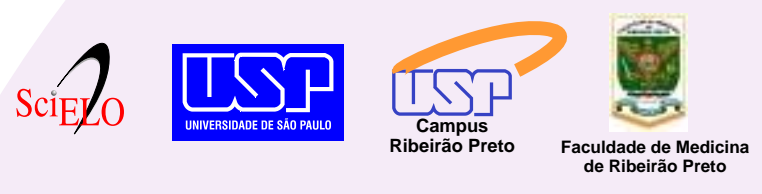

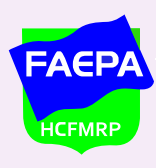

Ф SHIMADZU

GE Healthcare
Hotsite of proteomics metabolomics developped by:

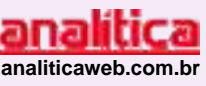

Thermo SCIENTIFIC 


\title{
The inhibitory effect of photodynamic therapy and of an anti-VCAM-1 monoclonal antibody on the in vivo growth of $\mathrm{C} 6$ glioma xenografts
}

\author{
Qi Zhan ${ }^{1}$, Wu Yue ${ }^{1}$ and Shaoshan $\mathrm{Hu}^{2}$ \\ ${ }^{1}$ Department of Neurosurgery, The Fourth Affiliated Hospital, ${ }^{2}$ Department of Neurosurgery, \\ The Second Affiliated Hospital, Harbin Medical University, Harbin, China
}

\begin{abstract}
We investigated the effect of photodynamic therapy (PDT) and of an anti-vascular cell adhesion molecule-1 (VCAM-1) monoclonal antibody on the in vivo growth of 66 glioma. Seven days after inoculation with C6 cells, adult male Wistar rats weighing 280-300 $\mathrm{g}$ with MRI-confirmed glioma were randomly assigned to 4 groups ( $\mathrm{N}=15$ per group): PDT + VCAM-1 antibody group; PDT group; VCAM-1 antibody group; control group. Eight days after inoculation, hematoporphyrin monomethyl ether (HMME) was administered as a photosensitizer and PDT was performed at $630 \mathrm{~nm}$ (illumination intensity: $360 \mathrm{~J} / \mathrm{cm}^{2}$ ) for $10 \mathrm{~min}$. VCAM-1 antibody (50 $\mu \mathrm{g} /$ $\mathrm{mL}$ ) was then administered $(0.5 \mathrm{~mL})$ through the tail vein every other day from day 8 to day 16 . At day 21,5 rats in each group were sacrificed and cancers were harvested for immunohistochemistry and Western blot assay for the detection of VCAM-1, and TUNEL assay was used to detect apoptosis. Survival and tumor volume were recorded in the remaining 10 rats in each group. In the PDT group, tumor growth was significantly suppressed (67.2\%) and survival prolonged (89.3\%), accompanied by an increase in apoptosis (369.5\%), when compared to control. Furthermore, these changes were more pronounced in the PDT + VCAM-1 antibody group. After PDT, VCAM-1 expression was markedly increased (121.8\%) and after VCAM-1 monoclonal antibody treatment, VCAM-1 expression was significantly reduced (58.2\%). PDT in combination with VCAM-1 antibody can significantly inhibit the growth of C6 glioma and prolong survival. This approach may represent a promising strategy in the treatment of glioma.
\end{abstract}

Key words: C6 glioma cells; Photodynamic therapy; Hematoporphyrin monomethyl ether; Anti-VCAM-1 monoclonal antibody; Apoptosis

\section{Introduction}

Glioma is a common intracranial malignancy accounting for approximately $40 \sim 50 \%$ of intracranial malignancies. The 5 -year survival rate is approximately $30 \%$ in patients with astrocytoma (1). Photodynamic therapy (PDT) has been an effective auxiliary strategy in the treatment of glioma (2-4). In PDT, either a photosensitizer or its metabolic precursor is administered to the patient. The target tissue is subjected to irradiation at specific wavelengths. When the photosensitizer and an oxygen molecule are in proximity, oxygen undergoes a chemical reaction that yields a reactive oxygen species. Through numerous signaling pathways, cell structures are damaged and cell functions are impaired, resulting in the death of cancer cells.

PDT with a lethal light dose can directly suppress the proliferation of cancer cells. However, PDT with a sublethal light dose may not only induce apoptosis of cancer cells, but activate signaling pathways related to cell survival, resulting in an escape response of cancer cells. This, in turn, compromises the efficacy of PDT and may lead to metastasis and recurrence of cancer (5). In addition, the energy density of light that deep tissues receive is relatively low due to the limited depth penetration of light used in PDT, and, therefore, the efficacy of PDT in deep cancer tissues is not favorable, often resulting in different PDT dose effects (6).

Vascular cell adhesion molecule-1 (VCAM-1) is a member of the immunoglobulin superfamily and its receptor is very late antigen-4 (VLA-4). VCAM-1 is poorly expressed in endothelial cells at their quiescent stage and in normal brain, but highly expressed in gliomas (7). In the serum of glioma patients, the sVCAM-1 level is also elevated, a fact that may be related to the invasiveness and angiogenesis of gliomas (8).

Correspondence: Wu Yue, 37 Yiyuan St, Nanggang District, Harbin 150086, China. Fax: +86-45-1825-53857.

E-mail: zhanqi51685168@163.com

Received November 30, 2010. Accepted April 5, 2011. Available online April 25, 2011. Published May 16, 2011. 
Hematoporphyrin monomethyl ether (HMME) is a novel photosensitive reagent developed in China. In vitro and in vivo studies (9-11) have shown that HMME is a promising photosensitizer. In the present study, the effect of HMMEmediated PDT and of VCAM-1 monoclonal antibody on the growth of glioma was investigated in order to explore their anti-angiogenesis effect on this tumor. We also studied VCAM-1 expression to better understand the mechanism of action of PDT on gliomas with the hope that our results may demonstrate the clinical application of PDT.

\section{Material and Methods}

\section{Cell culture}

The C6 glioma cell line was purchased from the Beijing Institute of Science, and cultured in RPMI 1640 medium (Gibco, USA) containing 10\% fetal bovine serum (Gibco) in an incubator containing $5 \% \mathrm{CO}_{2}$ at $37^{\circ} \mathrm{C}$.

\section{Rat C6 glioma model}

Inoculation of $\mathrm{C} 6$ cells was performed as previously described by Zelenkov et al. (12). Rats were anesthetized with $10 \%$ chloral hydrate ( $3 \mathrm{~mL} / \mathrm{kg}$; Sigma, USA) and then fixed in a stereotaxic instrument. After sterilization and skin incision, a hole was made in the skull $1.0 \mathrm{~cm}$ anterior to the anterior fontanel and $3.0 \mathrm{~cm}$ lateral to the sagittal suture. A syringe was inserted $5 \mathrm{~cm}$ into the cerebral cortex, and $10 \mu \mathrm{L}$ C6 glioma cell suspension ( $10^{6}$ cells) was injected. Injection was performed for $10 \mathrm{~min}$ and the syringe remained in the brain for $5 \mathrm{~min}$, followed by slow retraction. The hole was sealed with bone wax and the wound was closed.

\section{Grouping and treatment}

One week after inoculation, MRI was employed to detect cancer in the brains of the rats. A total of 60 male Wistar rats with gliomas, weighing 280-300 g (Beijing Vitalriver Experimental Animal Co., Ltd; License No. 046, China) were randomly assigned to 4 groups ( $N=15$ per group): PDT + VCAM-1 antibody group; PDT group; VCAM-1 antibody group; control group. Eight days after inoculation, PDT was carried out and $0.5 \mathrm{~mL}$ VCAM-1 monoclonal antibody (50 $\mu \mathrm{g} / \mathrm{mL}$; R\&D Systems, USA) was given every other day through the tail vein from day 8 to day $16(13,14)$. Rats in the control group and PDT group received saline $(0.5$ $\mathrm{mL}$ ). On day 21, 5 rats in each group were sacrificed and perfused transcardially with $4 \%$ paraformaldehyde and the brain was harvested for immunohistochemistry and Western blot assays. The remaining rats were used to determine survival rates. MRI was performed at both 2 and 3 weeks after inoculation to detect tumor volume and to calculate tumor growth inhibition rate. At the end of the experiment, animals were anesthetized and perfused transcardially with $4 \%$ paraformaldehyde (Sigma) and coronal sections were performed to observe the tumors. Tissues were processed for the Western blot assay and immunohistochemistry. All experimental procedures were approved by the Committee on Bioethics (Harbin Medical University).

\section{PDT treatment}

PDT was carried out as previously described (15). HMME (5 mg/kg; Fudan Zhangjiang Biotech Co., Ltd., China) was administered through the tail vein, and $3 \mathrm{~h}$ later animals were anesthetized with $10 \%$ chloral hydrate and fixed in a stereotaxic device, the skull was exposed, and a 1-cm diameter craniotomy was drilled over the right hemisphere $2.5 \mathrm{~cm}$ from the midline and $2.0 \mathrm{~cm}$ anterior to the bregma. Three minutes later, the inoculated tumor was exposed by a microneurosurgical method. The optical fiber of the PDT instrument (wavelength: $630 \mathrm{~nm}$; DIOMED630 Limited, UK) was placed on the tumor, followed by photodynamic treatment (light spot diameter: $1.1 \mathrm{~cm}$ ) for $10 \mathrm{~min}$. The treatment dose rates were $200 \mathrm{~mW} / \mathrm{cm}^{2}$, equivalent to $360 \mathrm{~J} / \mathrm{cm}^{2}$. After PDT, the hole was sealed with bone wax and the skin was sutured. Rats in the VCAM-1 antibody group and the control group did not receive HMME, but were subjected to all other procedures.

\section{MRI scanning}

MRI scanning was carried out using the Signa 3.0 T MRI System. The conditions for scanning were previously described (16): T1-weighted coronal and horizontal images were acquired from both unenhanced and enhanced scans. For enhanced scans, Gd-DTPA ( $0.4 \mathrm{mmol} / \mathrm{kg}$; Schering AG, Germany) was injected through the tail vein. The largest enhanced areas on the horizontal and coronal planes were analyzed and the maximum anteroposterior diameter $(\mathrm{L})$, width $(\mathrm{W})$ and height $(\mathrm{H})$ determined were used for the calculation of tumor volume $(\mathrm{V})$, as follows: $V=(4 / 3 \times \pi \times$ $\mathrm{L} \times \mathrm{W} \times \mathrm{H}) \times 1 / 8\left(\mathrm{~mm}^{3}\right)$.

\section{Western blot assay}

Total protein was extracted from $200 \mathrm{mg}$ frozen tissue and the protein concentration was determined. The protein was stored at $-70^{\circ} \mathrm{C}$ before use. Then, $20 \mu \mathrm{g}$ total protein was subjected to SDS-polyacrylamide gel $(6 \%$ resolving gel and 5\% stacking gel) electrophoresis, and transferred to PVDF membranes. The membranes were blocked with $5 \%$ non-fat milk in Tris-buffered saline and then treated with VCAM-1 antibody (1:500; Santa Cruz, USA) at $4^{\circ} \mathrm{C}$ overnight followed by incubation with horseradish peroxidase-conjugated secondary antibody (Santa Cruz) for $2 \mathrm{~h}$ at room temperature. Development was carried out with enhanced chemiluminescence reagents. $\beta$-actin was used as an internal reference.

\section{Immunohistochemistry}

Tissues were embedded in paraffin, cut into 5- $\mu \mathrm{m}$ sections and stained with H\&E. Sections were observed under a light microscope.

For immunohistochemistry, sections were deparaffinized 
and hydrated, followed by antigen retrieval. Endogenous peroxidase was inactivated with hydrogen peroxide. These sections were treated with rabbit anti-rat VCAM-1 antibody (1:400; Santa Cruz) or with rabbit anti-rat GFAP (glial fibrillary acidic protein) antibody (1:500; Santa Cruz). Immunohistochemistry was performed according to manufacturer instructions. Evaluation of sections was performed based on the proportion of positive cells: $<10 \%$, negative $(-) ; 11 \sim 30 \%$, weakly positive (+); 31 50\%, positive $(++)$; $>50 \%$, strongly positive $(+++)$. Sections were independently evaluated by two experienced pathologists who were blind to the conditions of the study. When the scores were different, a consensus was reached through consultation.

\section{Determination of apoptotic index by TUNEL assay}

The paraffin-embedded tissues were cut into sections and TUNEL assay was performed according to manufacturer instructions (Roche, Germany). TUNEL-positive cells demonstrated karyopyknosis, chromatin aggregation and a dark nucleus. Negative cells were blue and had a normal nucleus. A total of 20 fields were selected randomly from each section at a magnification of $400 \mathrm{X}$, positive cells were counted and the apoptotic index $(\mathrm{Al})$ was calculated as follows: $\mathrm{Al}=$ (number of positive cells $/$ total number of cells) $\times 100 \%$.

\section{Statistical methods}

Data are reported as means \pm SD. Statistical analyses were performed using the SPSS10.0 software. The survival data were analyzed by Kaplan-Meier survival analysis. Statistical comparisons of tumor volume, VCAM-1 expression, as well as numbers of apoptotic tumor cells between two different treatment groups, were made using $t$-tests. $\mathrm{P}<$ 0.05 was considered to be statistically significant.

\section{Results}

\section{H\&E staining, GFAP expression and MRI scanning}

H\&E staining showed invasive growth of the cancers. In addition, the nuclei of the cancer cells showed disintegration. Cancer cells invaded normal brain tissues without a clear boundary. Newly generated vessels were also noted.

Immunohistochemistry for GFAP revealed positive staining (brown granules) in a variety of cells, suggesting the presence of a glioma. A brain MRI scan revealed gliomas in the rat brain (Figure 1).
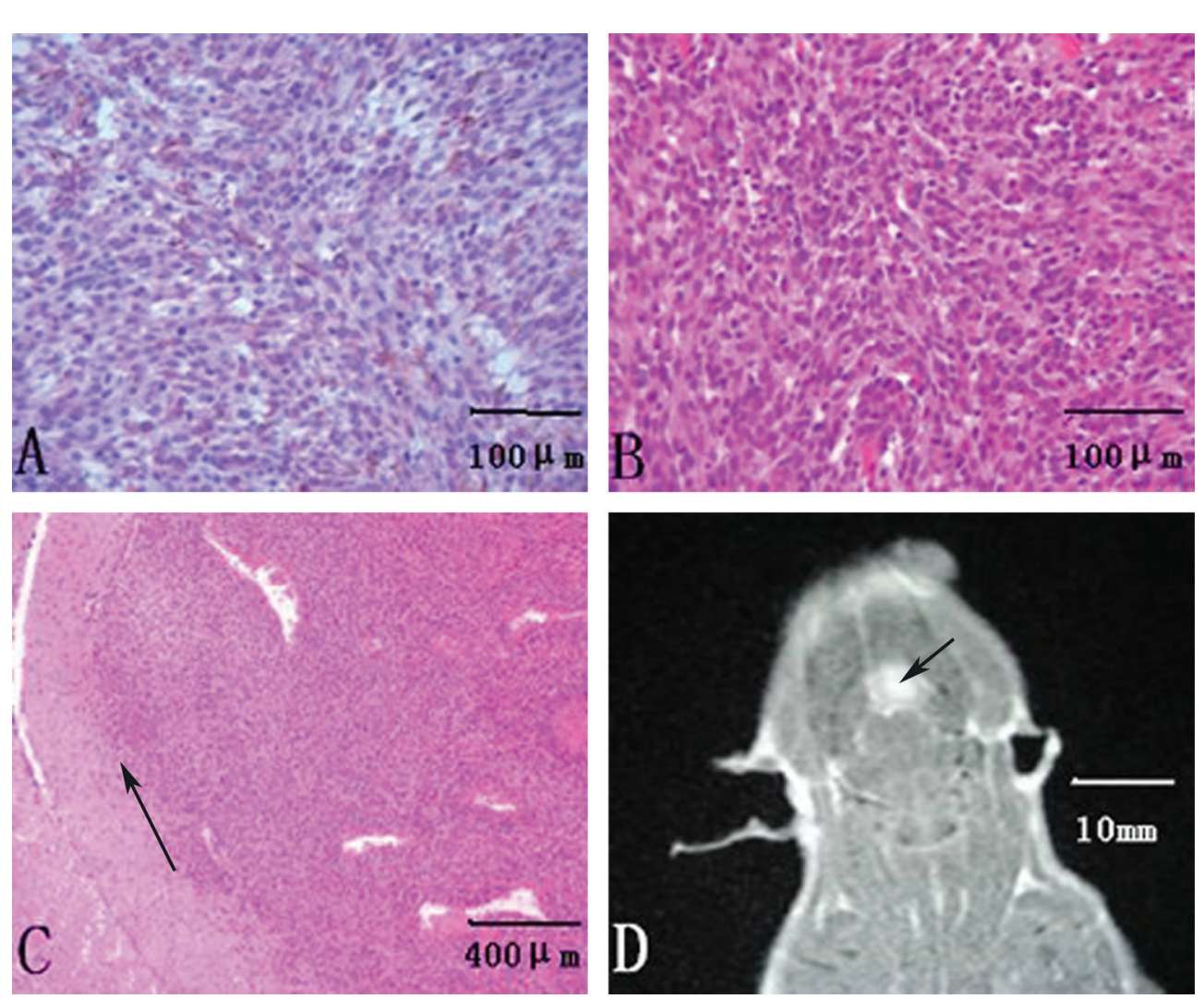

Figure 1. GFAP expression and representative photographs from MRI scanning. $A$, GFAP expression; $B$, H\&E staining; $C$, H\&E staining; the arrow shows the boundary between the cancer and normal brain tissue; $D$, the arrow on the rat MRI shows the cancer 1 week after inoculation. GFAP = glial fibrillary acidic protein. 


\section{Tumor volume and survival time}

One week after PDT treatment, tumor volume in the PDT group was markedly decreased compared to the control group $(P<0.05)$, suggesting decelerated cancer growth. In the VCAM-1 antibody group, tumor volume did not markedly change during the first week. However, 2 weeks after VCAM-1 antibody treatment, cancer growth was significantly slower compared to the control group $(\mathrm{P}<$ 0.05). In the PDT + VCAM-1 antibody group, cancer growth was significantly slower than in the control group or VCAM1 antibody group, but not different from the PDT group ( $P$ $>0.05$ ) during the first week. Two weeks after treatment, cancer growth of the PDT + VCAM-1 antibody group was remarkably slower than in the PDT group $(P<0.01)$. In the PDT + VCAM-1 antibody group, the tumor inhibition rate
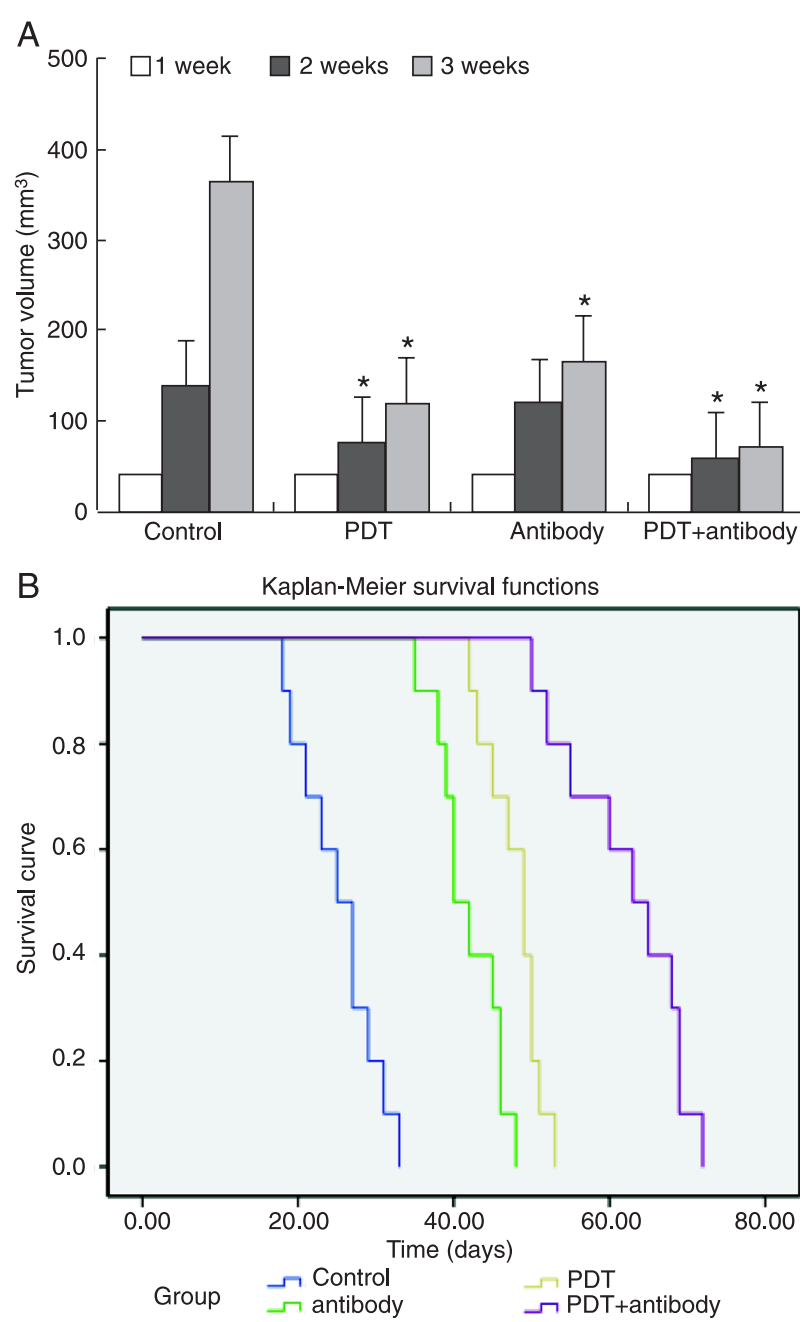

Figure 2. Tumor volume at 1,2 , and 3 weeks after inoculation (A) and Kaplan-Meier survival curves (B) in the 4 different groups. PDT = photodynamic therapy; antibody = VCAM-1 (vascular cell adhesion molecule-1). ${ }^{*} \mathrm{P}<0.05$ compared to the control group (two-sided paired $t$-test) was as high as $80.7 \%$, which was markedly higher than that of the PDT group (67.2\%) or VCAM-1 antibody group (54.6\%; Figure 2A).

Kaplan-Meier survival analysis showed that the mean survival was 25,300 \pm 1592 days for the control group, which was significantly shorter than that of the PDT group $(47,900 \pm 1130$ days $)$ or VCAM-1 antibody group $(41,900$ \pm 1329 days; $P<0.05)$. The survival in the PDT + VCAM-1 antibody group was the longest at $62,300 \pm 2450$ days ( $P$ $<0.01$; Figure 2B).

\section{Detection of protein expression of VCAM-1 by Western blot assay}

The protein expression of VCAM-1 is reported as the ratio of absorbance of the bands of VCAM-1 to that of $\beta$-actin. The expression of VCAM- 1 was $0.55 \pm 0.21$ in the control group, $1.22 \pm 0.45$ in the PDT group, $0.23 \pm 0.09$ in the VCAM-1 antibody group, and $0.68 \pm 0.26$ in the PDT + VCAM-1 antibody group. VCAM-1 expression in the PDT group was markedly higher compared to the control group $(\mathrm{P}<0.05)$. However, VCAM-1 expression in the VCAM-1 antibody group was markedly lower compared to the control group $(\mathrm{P}<0.05)$. Moreover, VCAM-1 expression in the PDT + VCAM-1 antibody group was lower compared to the PDT group $(P<0.05)$, but not different from the control group (P > 0.05; Figure 3).

\section{Immunohistochemistry for VCAM-1}

VCAM-1-positive cells contained brown granules.

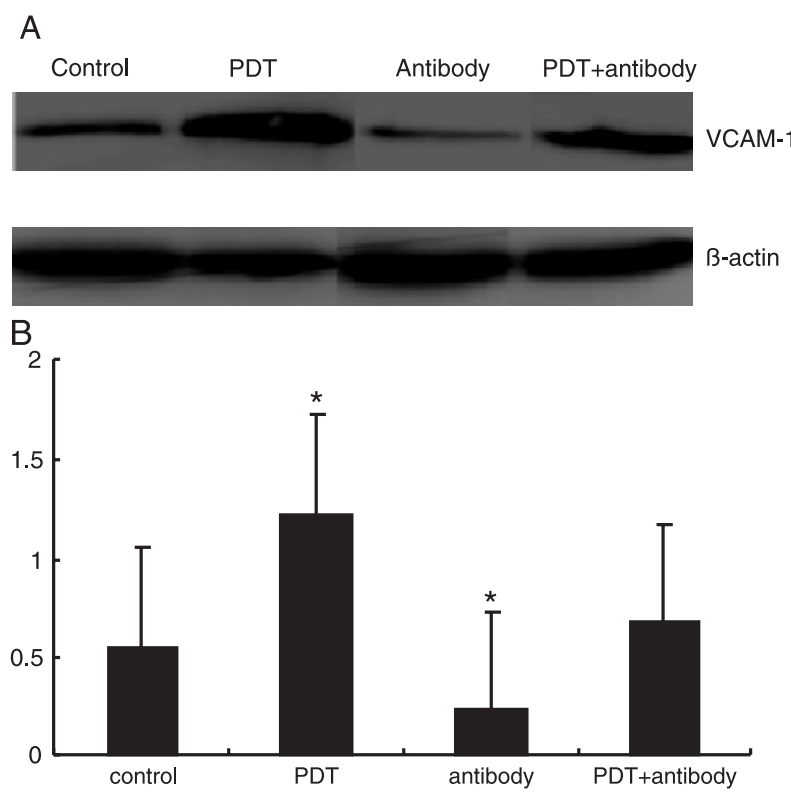

Figure 3. Western blot assay. A, Representative bands of VCAM1 and $\beta$-actin; $B$, expressions of VCAM- 1 normalized to $\beta$-actin for each of the 4 groups. PDT = photodynamic therapy; antibody $=$ VCAM-1 (vascular cell adhesion molecule-1). ${ }^{*} \mathrm{P}<0.05 \mathrm{com}-$ pared to the control group (two-sided paired $t$-test). 
VCAM-1 was mainly expressed in the cytoplasm and membrane of cancer cells and endothelial cells. VCAM-1 expression was markedly higher in the PDT group than in the control group, but was lower in the VCAM-1 antibody group compared to the control group. Moreover, VCAM-1 expression in the PDT + VCAM-1 antibody group was markedly lower compared to the PDT group, but not different from the control group ( $P>0.05$; Figure 4).

\section{Apoptotic index}

In the PDT group, VCAM-1 antibody group and PDT + VCAM-1 antibody group, the TUNEL assay showed that some cells had shrinkage and chromatin margination characterized by a crescent or lobular shape. Apoptotic bodies were noted. The Al was $39.3 \pm 3.4$, $25.4 \pm 2.8$, and $61.7 \pm 4.9 \%$ for the PDT group, VCAM-1 antibody group and PDT + VCAM-1 antibody group, respectively. In the control group, the cells showed integral morphology and scant apoptotic cells. The Al for the control group was $7.8 \pm$ $0.5 \%$ (Figure 5).

\section{Discussion}

Local invasion is the most important characteristic of glioma growth. Recurrence is frequently found in glioma patients and approximately $90 \%$ of recurrent gliomas are found only $2 \mathrm{~mm}$ away from the primary cancer (17). Therefore, removal of residual cancer cells is critical for the prevention of glioma recurrence. Our results showed that, after PDT, residual cancer cells highly expressed VCAM-1 (which is beneficial for angiogenesis) but that PDT in combination with VCAM-1 antibody significantly inhibited the growth of C6 gliomas.

Generally, the normal blood brain barrier may hinder the entry of photosensitizers into the brain. However, the blood brain barrier is damaged by cancer cells and is un-
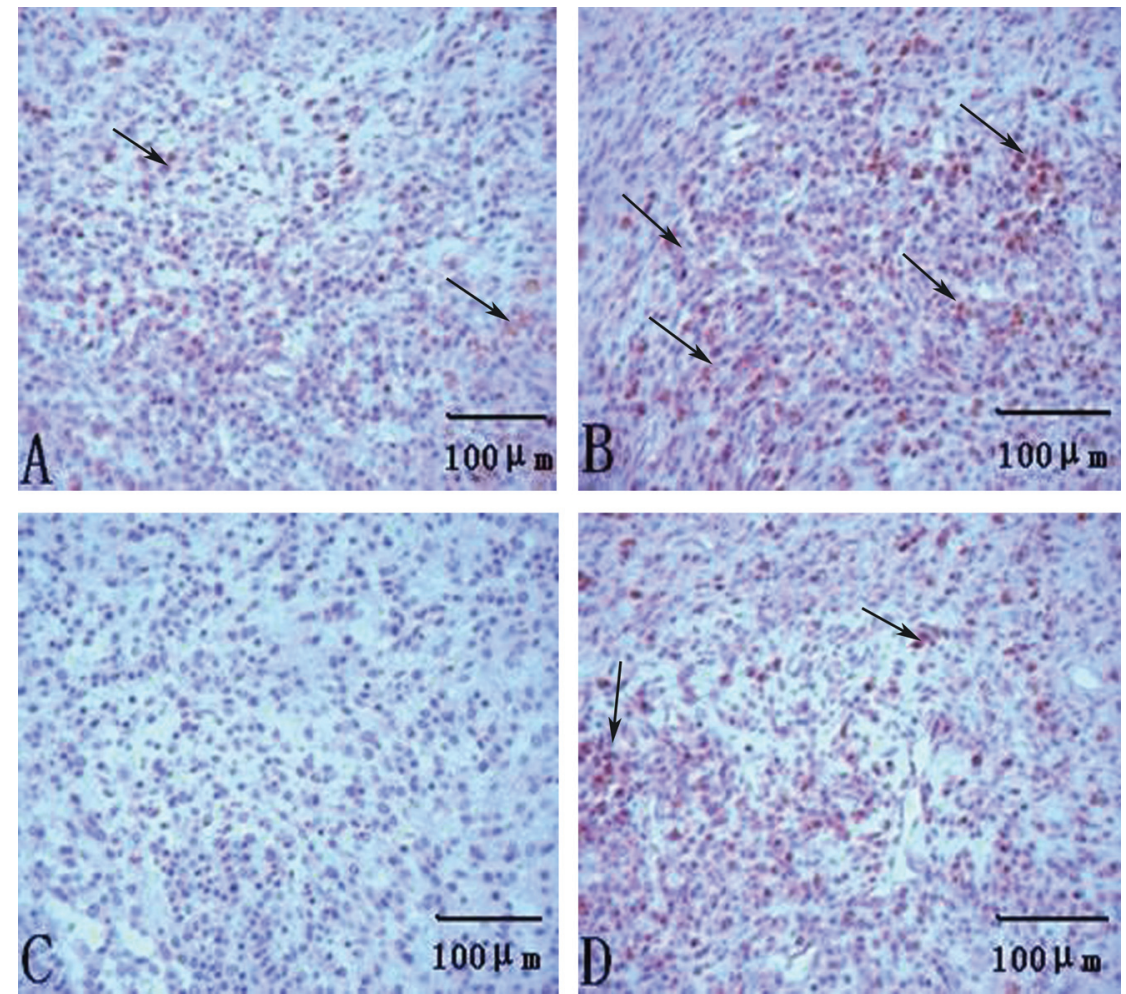

Figure 4. Immunohistochemistry for vascular cell adhesion molecule-1 (VCAM-1). A, Control group; $B$, photodynamic therapy (PDT) group; $C$, VCAM-1 antibody group; $D$, PDT + VCAM-1 antibody group. The arrows show the VCAM-1-positive cells.
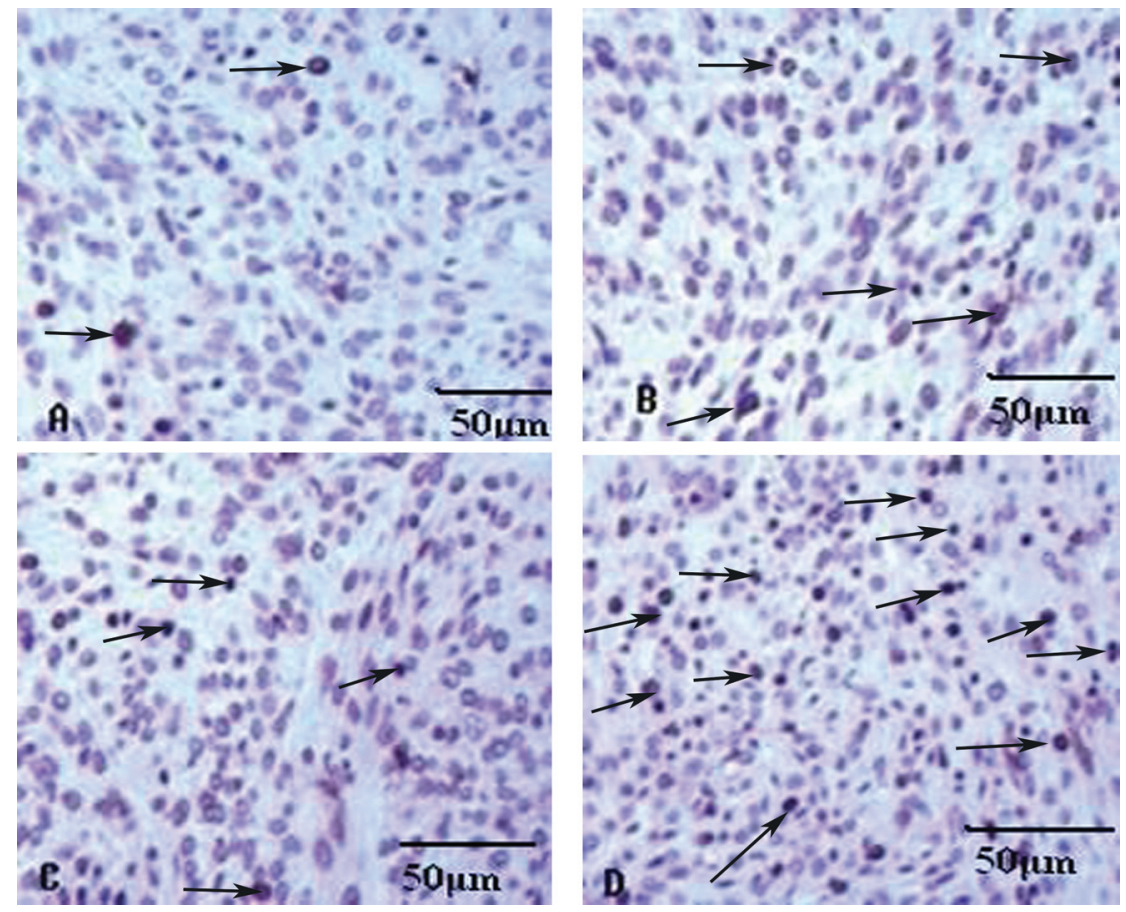

Figure 5. TUNEL assay. $A$, Control group; $B$, photodynamic therapy (PDT) group; $C$, VCAM-1 antibody group; $D$, PDT + VCAM-1 antibody group. Antibody = VCAM-1 (vascular cell adhesion molecule-1). The arrows show apoptotic cells. 
able to hinder the entry of photosensitizers. Thus, glioma cells have the ability to take up photosensitizers. In an in vitro study, $\mathrm{Hu}$ et al. (18) showed that HMME-mediated PDT could induce the apoptosis of C6 glioma cells and that calcium overload played an important role in extensive ultrastructural damage.

In the present study, the survival of the PDT group was significantly longer compared to the control group, which further confirmed that PDT can inhibit the growth of gliomas. Unfortunately, the energy density of light received by deep tissues is relatively low due to the limited light penetration depth used in PDT and, therefore, the efficacy of PDT in deep cancer tissues is not favorable, limiting its wider application

In the 1970's, Folkman et al. (19) first proposed the suppression of angiogenesis as an anti-tumor therapy. The growth and metastasis of solid vascular cancers, such as malignant gliomas, depend on angiogenesis, or newly generated blood vessels, for their blood supply. Since that time, the efficacy of anti-angiogenesis therapy has been confirmed in many clinical trials.

A variety of researchers have applied PDT in combination with anti-angiogenesis to the treatment of cancers with great success. Zhou et al. (20) showed that angiogenesis inhibitors (SU5416 and SU6668) in combination with PDT, when used in the treatment of mouse nasopharyngeal carcinoma, not only prolonged mouse survival but also inhibited cancer growth. Ferrario et al. (21) applied PDT to the treatment of mouse breast cancer. The results showed that vascular endothelial growth factor (VEGF) expression was increased after PDT but was markedly decreased after treatment with angiogenesis inhibitors (IM862 and EMAP-2). Bhuvaneswari et al. (22), using a mouse model, reported that anti-VEGF antibody (Bevacizumab) in combination with PDT was also effective in the treatment of human bladder cancer.

Kosharskyy et al. (23) applied an angiogenesis inhibitor (TNP-470) and PDT to the treatment of mouse prostate cancer and showed that PDT followed by treatment with TNP-470 was more effective than treatment with TNP-470 followed by PDT. This may be explained by the fact that TNP-470 treatment before PDT inhibits the angiogenesis of the cancer, preventing the photosensitizer from effectively entering the cancer and thus decreasing the efficacy of PDT. Bhuvaneswari et al. (24) showed that epidermal growth factor receptor (EGFR) expression was highly expressed after PDT. In addition, PDT followed by treatment with EGFR monoclonal antibody (Erbtux) not only inhibited the expression of EGFR but also reduced tumor volume and prolonged survival. Ferrario et al. $(25,26)$ indicated that cyclooxygenase-2 (COX-2) expression was markedly increased after PDT, influencing the efficacy of PDT. Yee et al. (27) applied a selective inhibitor of COX-2 (celecoxib) to enhance the efficacy of PDT in nasopharyngeal carcinoma and showed that the apoptosis of cancer cells was mark- edly increased, accompanied by inhibition of inflammatory angiogenesis, producing a favorable outcome.

However, the combined therapy with PDT and antiVCAM-1 antibody has not yet been applied to glioma management. Whether or not anti-VCAM-1 antibody can counter the up-regulated VCAM-1 expression and action induced by PDT and thereby increase the responsiveness of glioma to PDT has not been determined.

Our results showed that, after PDT, residual cancer cells highly expressed VCAM-1, which is beneficial for angiogenesis. Thus, cancer cells can escape the attack, with consequent proliferation of residual cancer cells and subsequent recurrence and metastases, which are the main cause of unfavorable long-term efficacy of PDT. Our study revealed that PDT in combination with VCAM-1 monoclonal antibody could markedly decrease PDT-induced VCAM-1 expression and simultaneously increase the apoptosis of cancer cells, which may be the primary way VCAM-1 monoclonal antibody enhances the efficacy of PDT in gliomas. However, the exact mechanism requires further study.

Additional studies (28) have revealed that VCAM-1 can induce angiogenesis by promoting the migration of endothelial cells to the angiogenic site with their subsequent proliferation. The relationship between VCAM-1 and angiogenesis has been confirmed in numerous cancer types. Hemmerlein et al. (29) synthesized a recombinant fusion protein targeting VCAM-1, aiming to block angiogenesis. This protein has been applied to the treatment of Hodgkin's lymphoma and small cell lung cancer. The results showed that this fusion protein could effectively inhibit angiogenesis, leading to coagulation and obstruction of the vascular system of cancers, with consequent cancer cell necrosis as well as delayed cancer growth. Garmy-Susini et al. (30) revealed that $\alpha 4 \beta 1-V C A M-1-m e d i a t e d$ adhesion between endothelial cells and pericytes was essential for the maturation of blood vessels. In addition, VCAM-1 antibody could inhibit the expressions of VEGF-A and basic fibroblast growth factor. Ding et al. (31) found that VCAM-1 expression was related to microvessel density in gastric cancer and might be involved in the angiogenesis and metastasis of gastric cancer. Maurer et al. (32) showed that the interaction between VCAM-1 and its ligand (VLA-4) could activate endothelial cells, promoting the metastasis of cancers. Duan et al. (33) found that, after ischemia, numerous adhesion molecules were expressed on endothelial cells where VCAM-1 expression was significantly elevated, and that VCAM-1 promoted the adhesion and aggregation of monocytes and facilitated the release of cytokines, resulting in angiogenesis. Hemmerlein et al. (29) found that tumor-associated macrophages were closely associated with VCAM-1 in renal cell carcinoma. Tumor-associated macrophages could promote the proliferation and invasion of cancer cells by secreting numerous cytokines, growth factors and/or matrix-degrading enzymes and were associated with angiogenesis in cancers (34). Shin et al. (35) found that the adhesion between gastric 
cancer cells and endothelial cells depended on VCAM-1 expression, in which caveolin-1 was involved. Caveolin-1 is highly expressed in endothelial cells in vivo and in vitro and closely related to angiogenesis (36).

Our results showed that anti-VCAM-1 monoclonal antibody could inhibit not only the endogenous VCAM-1 expression in normal $\mathrm{C} 6$ glioma cells but also the increased VCAM-1 expression induced by photodynamic therapy.

In this study, although PDT or antiangiogenic therapy alone indeed prolonged the survival time of the animals with gliomas, animals treated by the combined therapy had a significantly longer survival time than those receiving a single treatment modality. Concurrently, we found that the tumor response to this combination therapy was greater than the response to each treatment alone in terms of both direct inhibition of tumor growth and prevention of secondary tumor growth by inhibiting tumor angiogenesis and increasing tumor cell apoptosis. PDT alone may shrink or destroy tumors; however, it induces angiogenesis, which promotes tumor regrowth. Anti-angiogenic treatment inhibits the growth of

\section{References}

1. Smith JS, Chang EF, Lamborn KR, Chang SM, Prados MD, Cha $S$, et al. Role of extent of resection in the long-term outcome of low-grade hemispheric gliomas. J Clin Oncol 2008; 26: 1338-1345.

2. Hirschberg $\mathrm{H}$, Sun $\mathrm{CH}$, Krasieva $\mathrm{T}$, Madsen SJ. Effects of ALA-mediated photodynamic therapy on the invasiveness of human glioma cells. Lasers Surg Med 2006; 38: 939-945.

3. Madsen SJ, Kharkhuu K, Hirschberg H. Utility of the F98 rat glioma model for photodynamic therapy. J Environ Pathol Toxicol Oncol 2007; 26: 149-155.

4. Beck TJ, Kreth FW, Beyer W, Mehrkens JH, Obermeier A, Stepp $\mathrm{H}$, et al. Interstitial photodynamic therapy of nonresectable malignant glioma recurrences using 5-aminolevulinic acid induced protoporphyrin IX. Lasers Surg Med 2007; 39: 386-393.

5. Akita Y, Kozaki K, Nakagawa A, Saito T, Ito S, Tamada Y, et al. Cyclooxygenase-2 is a possible target of treatment approach in conjunction with photodynamic therapy for various disorders in skin and oral cavity. Br J Dermatol 2004; 151: $472-480$.

6. Zhu TC, Hahn SM, Kapatkin AS, Dimofte A, Rodriguez CE, Vulcan TG, et al. In vivo optical properties of normal canine prostate at $732 \mathrm{~nm}$ using motexafin lutetium-mediated photodynamic therapy. Photochem Photobiol 2003; 77: 81-88.

7. Maenpaa A, Kovanen PE, Paetau A, Jaaskelainen J, Timonen T. Lymphocyte adhesion molecule ligands and extracellular matrix proteins in gliomas and normal brain: expression of VCAM-1 in gliomas. Acta Neuropathol 1997; 94: 216-225.

8. Salmaggi A, Eoli M, Frigerio S, Ciusani E, Silvani A, Boiardi A. Circulating intercellular adhesion molecule-1 (ICAM-1), vascular cell adhesion molecule-1 (VCAM-1) and plasma thrombomodulin levels in glioblastoma patients. Cancer Lett 1999; 146: 169-172. tumor cells by cutting off the tumor's nutritional supply. Our data showed that the combined therapy provided additive anticancer efficacy in that the angiogenesis induced by PDT was counteracted by the antiangiogenic agents.

The combined therapy can more effectively inhibit cancer growth, prolong survival, and increase the apoptosis of cancer cells compared to either PDT or VCAM-1 monoclonal antibody alone. Therefore, the combination treatment may be a reasonable strategy to improve the clinical outcome of glioblastomas. Future experiments involving clinical gliomas can be performed, first using surgical resection and then using combination therapy with local invasion by killer cells. Medical intervention targeted on VCAM-1 in the process of photodynamic treatment has an optimistic prospect.

\section{Acknowledgments}

Research supported by the National Natural Science Foundation of China (No.30470586).

9. Ding X, Xu Q, Liu F, Zhou P, Gu Y, Zeng J, et al. Hematoporphyrin monomethyl ether photodynamic damage on HeLa cells by means of reactive oxygen species production and cytosolic free calcium concentration elevation. Cancer Lett 2004; 216: 43-54.

10. Song K, Kong B, Qu X, Li L, Yang Q. Phototoxicity of Hemoporfin to ovarian cancer. Biochem Biophys Res Commun 2005; 337: 127-132.

11. Wang JB, Liu LX, Pan SH, Wang CY, Fu QF. Therapeutic effect of photodynamic therapy using hematoporphyrin monomethyl ether (HMME) on human cholangiocarcinoma cell line QBC939. Neoplasma 2010; 57: 79-85.

12. Zelenkov P, Baumgartner R, Bise K, Heide M, Meier R, Stocker $S$, et al. Acute morphological sequelae of photodynamic therapy with 5-aminolevulinic acid in the C6 spheroid model. J Neurooncol 2007; 82: 49-60.

13. Slack-Davis JK, Atkins KA, Harrer C, Hershey ED, Conaway $M$. Vascular cell adhesion molecule-1 is a regulator of ovarian cancer peritoneal metastasis. Cancer Res 2009; 69: 1469-1476

14. Justicia C, Martin A, Rojas S, Gironella M, Cervera A, Panes $\mathrm{J}$, et al. Anti-VCAM-1 antibodies did not protect against ischemic damage either in rats or in mice. $J$ Cereb Blood Flow Metab 2006; 26: 421-432.

15. Schreiber S, Gross S, Brandis A, Harmelin A, RosenbachBelkin V, Scherz A, et al. Local photodynamic therapy (PDT) of rat C6 glioma xenografts with Pd-bacteriopheophorbide leads to decreased metastases and increase of animal cure compared with surgery. Int J Cancer 2002; 99: 279-285.

16. Ulmer S, Reeh M, Krause J, Herdegen T, Heldt-Feindt J, Jansen $\mathrm{O}$, et al. Dynamic contrast-enhanced susceptibilityweighted perfusion MRI (DSC-MRI) in a glioma model of the rat brain using a conventional receive-only surface coil with a inner diameter of $47 \mathrm{~mm}$ at a clinical 1.5 T scanner. $J$ 
Neurosci Methods 2008; 172: 168-172.

17. Burton EC, Prados MD. Malignant gliomas. Curr Treat Options Oncol 2000; 1: 459-468.

18. Hu SS, Cheng HB, Zheng YR, Zhang RY, Yue W, Zhang $\mathrm{H}$. Effects of photodynamic therapy on the ultrastructure of glioma cells. Biomed Environ Sci 2007; 20: 269-273.

19. Folkman J. Anti-angiogenesis: new concept for therapy of solid tumors. Ann Surg 1972; 175: 409-416.

20. Zhou Q, Olivo M, Lye KY, Moore S, Sharma A, Chowbay B. Enhancing the therapeutic responsiveness of photodynamic therapy with the antiangiogenic agents SU5416 and SU6668 in murine nasopharyngeal carcinoma models. Cancer Chemother Pharmacol 2005; 56: 569-577.

21. Ferrario A, von Tiehl KF, Rucker N, Schwarz MA, Gill PS, Gomer CJ. Antiangiogenic treatment enhances photodynamic therapy responsiveness in a mouse mammary carcinoma. Cancer Res 2000; 60: 4066-4069.

22. Bhuvaneswari R, Yuen GY, Chee SK, Olivo M. Hypericinmediated photodynamic therapy in combination with Avastin (bevacizumab) improves tumor response by downregulating angiogenic proteins. Photochem Photobiol Sci 2007; 6: 1275-1283.

23. Kosharskyy B, Solban N, Chang SK, Rizvi I, Chang Y, Hasan $\mathrm{T}$. A mechanism-based combination therapy reduces local tumor growth and metastasis in an orthotopic model of prostate cancer. Cancer Res 2006; 66: 10953-10958.

24. Bhuvaneswari R, Gan YY, Soo KC, Olivo M. Targeting EGFR with photodynamic therapy in combination with Erbitux enhances in vivo bladder tumor response. Mol Cancer 2009; 8: 94.

25. Ferrario A, Von Tiehl K, Wong S, Luna M, Gomer CJ. Cyclooxygenase-2 inhibitor treatment enhances photodynamic therapy-mediated tumor response. Cancer Res 2002; 62: 3956-3961.

26. Ferrario A, Fisher AM, Rucker N, Gomer CJ. Celecoxib and NS-398 enhance photodynamic therapy by increasing in vitro apoptosis and decreasing in vivo inflammatory and angiogenic factors. Cancer Res 2005; 65: 9473-9478.
27. Yee KK, Soo KC, Olivo M. Anti-angiogenic effects of Hypericin-photodynamic therapy in combination with Celebrex in the treatment of human nasopharyngeal carcinoma. Int $J$ Mol Med 2005; 16: 993-1002.

28. Koch AE, Halloran MM, Haskell CJ, Shah MR, Polverini PJ. Angiogenesis mediated by soluble forms of E-selectin and vascular cell adhesion molecule-1. Nature 1995; 376: 517519.

29. Hemmerlein B, Scherbening J, Kugler A, Radzun HJ. Expression of VCAM-1, ICAM-1, E- and P-selectin and tumour-associated macrophages in renal cell carcinoma. Histopathology 2000; 37: 78-83.

30. Garmy-Susini B, Jin H, Zhu Y, Sung RJ, Hwang R, Varner J. Integrin alpha4beta1-VCAM-1-mediated adhesion between endothelial and mural cells is required for blood vessel maturation. J Clin Invest 2005; 115: 1542-1551.

31. Ding YB, Chen GY, Xia JG, Zang XW, Yang HY, Yang L. Association of VCAM-1 overexpression with oncogenesis, tumor angiogenesis and metastasis of gastric carcinoma. World J Gastroenterol 2003; 9: 1409-1414.

32. Maurer CA, Friess H, Kretschmann B, Wildi S, Muller C, Graber $\mathrm{H}$, et al. Over-expression of ICAM-1, VCAM-1 and ELAM-1 might influence tumor progression in colorectal cancer. Int J Cancer 1998; 79: 76-81.

33. Duan $\mathrm{H}$, Cheng L, Sun $\mathrm{X}, \mathrm{Wu} \mathrm{Y}$, Hu L, Wang J, et al. LFA-1 and VLA-4 involved in human high proliferative potentialendothelial progenitor cells homing to ischemic tissue. Thromb Haemost 2006; 96: 807-815.

34. Lewis CE, Pollard JW. Distinct role of macrophages in different tumor microenvironments. Cancer Res 2006; 66: 605-612.

35. Shin J, Kim J, Ryu B, Chi SG, Park H. Caveolin-1 is associated with VCAM-1 dependent adhesion of gastric cancer cells to endothelial cells. Cell Physiol Biochem 2006; 17: 211-220.

36. Lin MI, Yu J, Murata T, Sessa WC. Caveolin-1-deficient mice have increased tumor microvascular permeability, angiogenesis, and growth. Cancer Res 2007; 67: 2849-2856. 